\title{
La photographie, déclencheur de parole en classe de FLE : la fiction dans les interactions
}

\author{
Catherine Muller \\ Université Paris 3, DILTEC \\ catherine.muller9@gmail.com
}

\section{Objet de recherche et méthodologie}

La didactique des langues étrangères, dans ses dimensions méthodologiques, a entretenu et entretient encore un rapport étroit avec les images. En effet, des supports visuels sont utilisés en cours de langue pour activer la réception écrite et orale, ainsi que la production écrite et orale. Notre recherche porte sur l'utilisation de la photographie d'art en classe de langue pour déclencher la parole et l'interaction.

Pour cela, une observation participante a été menée dans des classes de français enseigné comme langue étrangère à Paris avec deux groupes de niveau B1-B2 (groupes A et B). Des photographies d'art ont été montrées à des apprenants originaires de différentes aires culturelles (Amérique latine, Moyen-Orient, Asie, Europe de l'Est). Ces images ont été sélectionnées pour leur ambiguïté, leur capacité à intriguer et à faire parler. Il s'agit des photographies ${ }^{1}$ : Les mariés de A. Tress (SEQ 1), Kisangani, Zaïre- Congo de R. Lemoyne (SEQ 2), Nina et Julie - Trouville de D. Issermann (SEQ 3), Honor Frazer de D. Issermann (SEQ 4) et Excited man de R. Ballen (SEQ 5). L'enseignante ne fournit pas d'indications sur la signification des photos, elle n'enseigne pas l'analyse de l'image et ne guide pas l'interprétation. Ces séquences ne sont pas en lien avec le reste du cours. La seule compétence activée est l'interaction orale.

Cette recherche est en continuité avec la réflexion sur les méthodologies en didactique des langues. Elle rejoint une problématique d'enseignement classique : comment faire parler les apprenants ? Dans l'approche par compétences, les apprenants sont exposés à des échantillons d'interactions, qui constituent « des exemples des productions visées » (Beacco 2007 : 147). L'activité mise en œuvre prend le contrepied de cette conception puisqu'elle consiste à ne pas fournir d'input discursif. L'enseignante montre seulement une photographie dont la force déclenche une verbalisation. Il s'agit d'une autre forme possible d'entraînement à l'interaction. Ici, l'interaction est produite par contrainte.

Celle-ci s'orchestre selon deux scénarios. Dans l'un, la dynamique est portée par le groupe. Les apprenants interagissent de manière relativement autonome et sont capables de faire tourner la parole. Mais des impasses peuvent se créer par manque d'éléments ou en raison de difficultés linguistiques. Les tours de parole gérés par le groupe s'épuisent et l'enseignant relance alors le débat par des questions : il s'agit du deuxième scénario. Ce phénomène est évoqué en ces termes par C. de Margerie : «Puis, au moment où tout le monde s'est arrêté de parler, je propose de légender ce collage et les propositions repartent »(2007: 104). L'enseignant joue ainsi le rôle de médiateur et n'apparaît plus comme le détenteur de la norme.

L'analyse du corpus porte sur la forme et les enjeux des interactions déclenchées par les photographies, ainsi que sur les perspectives didactiques apportées par une telle activité. Nous proposons ici d'aborder plus spécifiquement le recours aux créations fictionnelles par les apprenants. Les particularités de l'énonciation seront mises en évidence.

L'interprétation de la photo par les apprenants peut se caractériser par des constructions narratives. La présence de récit est soulignée par $\mathrm{C}$. de Margerie qui considère que l'image est « histoire, enfin une ou plusieurs d'ailleurs, que chacun est libre de raconter à sa manière » (2006: 107). Mais le récit prend également la forme de la fiction avec une pseudo-référence au niveau des pronoms personnels : le " je », le « nous », le « vous » sont employés pour référer à une situation imaginaire. Il s'agit d'un exemple de polyphonie énonciative, phénomène récurrent de la classe de langue analysé par F. Cicurel (1996). Si l'entrée dans la fiction est mise en évidence par des marques de l'irréel, certains exemples témoignent 
aussi d'une instabilité énonciative. Dans notre corpus, ces incursions dans le monde imaginaire s'expliquent par la spécificité de l'image. La créativité des apprenants peut s'exprimer grâce au pouvoir évocateur des photos. Ainsi, la photographie semble propice à la verbalisation de mondes imaginaires.

Dans un premier temps seront présentées les constructions fictionnelles en classe de langue. On définira ensuite la polyphonie énonciative selon O. Ducrot, avant d'identifier les circonstances propices à l'apparition de la polyphonie et de la fiction. Enfin, les perspectives didactiques seront mises en évidence.

\section{Les constructions fictionnelles en classe de langue}

Si le corpus comporte une dimension narrative énoncée à la troisième personne, les apprenants manifestent également une propension à la fiction où ils ont recours à la première personne pour parler d'un autre locuteur qu'eux. D. Coste souligne que la classe constitue « un lieu de fictionnalisation » avec « un contact d'ordre en partie imaginaire avec la langue » (1991:247).

La présence de la fiction dans la classe de langue a été mise en évidence par F. Cicurel. Il s'agit d'un phénomène de «pseudo-référence » (Cicurel 1996 : 81). La fiction constitue l'une des caractéristiques du discours de la classe : "l'instauration d'un contrat de fiction lorsqu'il y a nécessité de configurer des univers imaginaires pour faire vivre la langue » (Cicurel $2002: 3$ ). L'auteur explique ainsi la présence de la fiction: "Comme enseignant et apprenants doivent à tout prix communiquer - pour apprendre/enseigner la langue - ils vont s'inventer des rôles fictionnels » (Cicurel 1985: 8). Ainsi, la fiction est envisagée comme un moyen de communiquer et d'éviter les restrictions liées à une «énonciation réelle ». Assumer une identité fictive permet une variété de «lieux communicationnels» (Cicurel $2003: 1$ ).

La fiction se caractérise par des marques de l'irréel. Les participants jouent à être, ils font « comme si ». Il s'agit de locuteurs virtuels et la parole est imaginaire. La fiction autorise des « discours simulés par lesquels les énonciateurs font semblant d'avoir besoin de quelque chose ou d'être quelqu'un d'autre qu'eux-mêmes » (Cicurel 1985 : 16). F. Cicurel fait figurer « des répétitions de discours d'autrui, des jeux de simulation, des jeux de rôle » (1985: 16) dans les exemples de fiction. D. O. Soares da Rocha compare la fiction dans la classe de langue au théâtre et souligne « la dimension de théâtralisation du langage qu[e la classe de langue] tend à développer chez ses participants » (1996:61).

On s'interrogera sur la particularité de la fiction lorsqu'elle est déclenchée par la présence de photographies. L'enseignante n'est pas à l'origine de la fiction : ce sont les apprenants qui introduisent une parole imaginaire à partir des images. On s'éloigne alors de la conception selon laquelle l'enseignant théâtralise et met en scène. Par exemple, D. O. Soares da Rocha considère que le professeur « devient une sorte de metteur en scène, une "voix off» qui dirige la scène, corrige les acteurs, évalue leurs performances » (1996: 63). Dans les interactions que nous analysons, la fiction n'apparait pas dans les stratégies d'explication de l'enseignante, mais dans l'expression même des apprenants qui inventent une histoire. Ils font ainsi parler les personnages. L'enseignante ne fournit pas de consignes explicites comme « imaginez que ». Elle leur propose seulement d'attribuer un titre aux photos. Les apprenants peuvent s'exprimer à partir des images par l'intermédiaire de la fiction.

La distinction entre la narration et la fiction repose sur l'énonciation. La fiction est caractérisée par des marques de la polyphonie : les pronoms de la première et de la deuxième personnes sont employés sans se référer au sujet parlant ou à l'auditeur. Un premier exemple permettra de distinguer la fiction de la narration simple : 


\author{
SEQ $1 \mathrm{~B}^{2}$ : \\ 191 Jawad voilà l'autre + il jure devant le juge parce qu'il va accepter son \\ son mariage avec cette femme
}

\author{
SEQ 1A : \\ 010 Djibril
}

ah (éclats de rire) ça c'est très joli euh + vous avez fait du doublage des photos + des photos mariage + je jure euh : je vais être fidèle à elle bon

Dans ces deux extraits, les apprenants ont été exposés à la photo Les mariés d'A. Tress. Dans la séquence $1 \mathrm{~B}$, Jawad décrit le personnage et fait le récit de la scène. Il emploie la troisième personne pour se référer au personnage : «il jure », « il va accepter ». En revanche, dans la séquence 1A, Djibril utilise la première personne pour parler du personnage. Le « je » ne réfère pas à Djibril, mais au marié de la photo.

\title{
3 La polyphonie énonciative
}

La fiction se manifeste au niveau de l'énonciation et plus particulièrement des pronoms personnels. On peut ainsi repérer des observables : «Qui est le je qui parle ? A qui s'adresse-t-il ? De quel objet parle-til ? Quelle est la place d'un discours simulé ? Y a-t-il des marques d'une énonciation non complètement assumée par le locuteur? » (Cicurel 1996: 77). Après avoir défini la polyphonie chez O. Ducrot, des exemples de pseudo-référence et de virtualité seront analysés.

\subsection{La polyphonie selon O. Ducrot}

La polyphonie permet de rendre compte de la virtualité des énonciateurs et des phénomènes de pseudoréférence. Elle a été étudiée par O. Ducrot dans les échanges quotidiens. Dans Les mots du discours (1980 : 43-44), il distingue le locuteur qui est l'«auteur des paroles» des énonciateurs qui sont les « agents des actes illocutionnaires ». De la même manière, il oppose l'allocutaire, qui est l'« être à qui les paroles sont dites » aux destinataires qui sont « les patients des actes ». P. Larcher (1998), qui analyse la théorie d'O. Ducrot, met en évidence les différences entre ses écrits de 1980 et de 1984. Il définit ainsi sa théorie de 1980 : « Sous sa forme primitive, la polyphonie consiste essentiellement à poser l'existence de deux couples de personnages : d'une part le locuteur, responsable de l'énonciation et qui a pour pendant l'allocutaire (objet de l'énonciation) et d'autre part l'énonciateur, responsable de l'acte illocutoire et qui a pour pendant le destinataire (objet ou patient de l'acte illocutoire) » $(1998: 215)$. Selon cette conception, lorsqu'un apprenant dit «je » pour se référer au personnage sur la photo, le locuteur correspond à l'apprenant et l'énonciateur au personnage.

Dans Le dire et le dit (1984) au chapitre 8, O. Ducrot reformule sa théorie. Il distingue trois instances : le sujet parlant, le locuteur et l'énonciateur. Le sujet parlant est l'individu qui produit l'énoncé. Il s'agit d'un «élément de l'expérience » (1984: 199), d'un « être empirique » (ibid.). L'auditeur constitue le pendant du sujet parlant. Le locuteur, quant à lui, est responsable de l'énoncé. Il peut mettre en scène des énonciateurs. Le narrateur constitue son équivalent littéraire (1984: 207). L'allocutaire lui fait face. O. Ducrot distingue ensuite le locuteur en tant que tel L, «source de l'énonciation » (1984: 201), qui est seulement responsable de l'énoncé, du locuteur en tant qu'être du monde $\lambda$. Ce dernier est entre autres responsable de l'énoncé, mais il s'agit d'une personne complète qui est «objet de l'énonciation » (1984: 201). Enfin, «l'énonciateur est au locuteur ce que le personnage est à l'auteur » (1984:205). Il est défini par son point de vue : c'est «la personne du point de vue de laquelle les événements sont présentés » (1984 : 208). O. Ducrot précise : «le locuteur, responsable de l'énoncé, donne existence, au moyen de celui-ci, à des énonciateurs dont il organise les points de vue et les attitudes » (1984:205). L'énonciateur a pour pendant le destinataire. O. Ducrot fournit principalement des exemples où il distingue le locuteur de l'énonciateur dans le cas de la négation. Dans notre corpus, l'énonciateur n'apparaît pas de façon aisée. 
Selon cette théorie, l'apprenant peut donc être assimilé au sujet parlant et le locuteur au personnage sur la photo. Pour résumer, O. Ducrot montre que derrière un « je » peut se cacher un autre «je »: «plusieurs voix parlent spontanément » $(1984: 171)$.

Pour analyser la polyphonie dans le corpus, on considèrera les différentes sources énonciatives dans le discours des apprenants. On s'appuiera sur l'étude des pronoms personnels en se demandant à qui l'énoncé est adressé et à qui les pronoms personnels renvoient. Quand l'apprenant dit « je », il peut se référer à lui-même en tant qu'individu ou qu'apprenant, à un «je » générique ou à un personnage de l'image. F. Cicurel précise que l'apprenant peut être «l'énonciateur d'un énoncé dont il n'est pas l'auteur» (1996: 80). En distinguant l'énonciateur de l'auteur, c'est-à-dire du sujet parlant, elle ne fait pas référence au locuteur de la théorie d'O. Ducrot. D. O. Soares da Rocha précise qu' « une constante fluctuation de l'identité de ses acteurs » $(1996: 62)$ est caractéristique du discours de la classe de langue.

\subsection{Polyphonie et impératif}

Avec les pronoms personnels « on » et "vous », «le locuteur introduit un sujet-énonciateur, qui est supposé être l'agent de la scène » (Cicurel 1991: 267), ce qui implique l'auditoire. L'analyse de la polyphonie commencera par l'étude de l'emploi de l'impératif chez les apprenants à partir de deux séquences :

\begin{tabular}{|c|c|c|}
\hline \multicolumn{2}{|c|}{ SEQ 2A : } & \multirow[b]{2}{*}{$\begin{array}{l}\text { ça c'est pas prier peut-être euh viens difficile tout de suite dono } \\
\text { maintenant oh Dieu quelque chose comme ça }\end{array}$} \\
\hline & Roshan & \\
\hline 231 & Roshan & $\begin{array}{l}\text { c'est possible parce que tu vois en Afrique ? voilà aujourd'hui } \\
\text { comme ça mais là-bas ? deux cents ans en Afrique xxxx parce }\end{array}$ \\
\hline & & 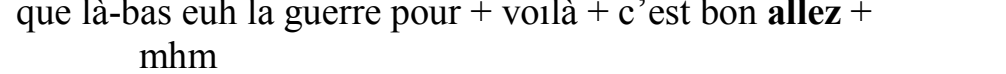 \\
\hline & Roshar & $\begin{array}{l}\text { restez calme }+ \text { comment on dit }+ \text { c'est } ?+\text { euh }:++ \text { c'est } ?++ \\
\text { la liberté la liberté la liberté }+ \text { arrêter la guerre }\end{array}$ \\
\hline
\end{tabular}

SEQ 2B :

278 Luiza

AIDEZ-NOUS

$279 \mathrm{P}$

aidez-nous ? ouais ? ouais ? + si vous deviez donner un titre + ce serait...?

\begin{tabular}{|c|c|c|}
\hline 30 & \multicolumn{2}{|c|}{ Abdel à l'aide } \\
\hline 281 & Rumalda & il faut partager \\
\hline 82 & $\mathrm{P}$ & il faut partager? ouais? \\
\hline 33 & Abdel à & avis c'est à l'aide \\
\hline 4 & $\mathrm{P}$ & à l'aide? oui ? ouais? d'autres idées de titre? \\
\hline & Carolina & soyez compassifs \\
\hline
\end{tabular}

Dans la séquence 2A, la première occurrence d'impératif par Roshan apparaît à la deuxième personne du singulier : « viens » (098). Ce tour de parole fournit peu d'indications sur l'allocutaire. En revanche, dans les deux autres emplois de l'impératif : « allez » (231) et « restez » (233), Roshan semble s'adresser aux enfants présents sur la photographie Kisangani Zaïre-Congo. Plus précisément, le sujet parlant Roshan, par la voix du locuteur, personnage présent dans le hors-champ, s'adresse aux enfants de la photo. Dans la séquence $2 \mathrm{~B}$, les apprenants ont été exposés à la même photographie. Le locuteur du premier énoncé à l'impératif ( « AIDEZ-NOUS » en 278) semble être les enfants de la photographie. Il s'agit d'un locuteur collectif, comme le montre la marque du pluriel «NOUS». Cet énoncé peut être adressé à des personnages placés en hors-champ, comme le photographe, ou bien aux spectateurs de la photo. En 279, l'enseignante reprend l'énoncé de Luiza de façon autonymique : « aidez-nous ? ». Elle s'adresse à Luiza. 
Pour cela, elle reprend les paroles de l'apprenante qui lui étaient adressées, mais se référaient également aux personnages du hors-champ. Il s'agit d'un exemple d'instabilité énonciative.

Le tour de parole de Luiza en 278 rejoint la proposition de titre d'Abdel en 280 et 283 : «à l'aide». Ici encore, on remarque plusieurs régimes discursifs. En effet, l'apprenant Abdel, sujet parlant, répond à la sollicitation de l'enseignante en 279 ( «si vous deviez donner un titre »). Abdel est également le locuteur «auteur des paroles» (Ducrot 1980: 43). Cependant, cette proposition constitue un discours direct rapporté qui peut être attribué aux enfants présents sur la photo. Les garçons de l'image correspondent donc aux énonciateurs mis en scène par le locuteur. L'énoncé d'Abdel est également adressé à différentes instances. Premièrement, l'ensemble de la classe constitue l'auditeur. L'allocutaire est plus particulièrement l'enseignante à qui l'apprenant répond. Enfin, le destinataire est composé d'une part des témoins de la scène et d'autre part des spectateurs de la photographie.

Pour analyser le tour de parole de Carolina en 285 «soyez compassifs », on s'appuie sur «la distinction entre l'allocutaire (c'est-à-dire la personne à qui l'énonciation est censée être adressée) et le destinataire (c'est-à-dire la personne censée être l'objet des actes illocutoires) » établie par O. Ducrot (1980:233). L'allocutaire est composé des participants de la classe, c'est-à-dire les apprenants et l'enseignante. Les destinataires sont ceux qui voient la photographie. En effet, il semble que l'énonciateur du message « soyez compassifs » soit davantage le photographe que les enfants de la photo. L'enseignante a demandé aux apprenants d'attribuer un titre à la photographie, tâche revenant souvent au photographe.

\subsection{Valeur spécifique, valeur générique et virtualité}

Le corpus comporte une récurrence de pronoms « on » et «nous » dans le discours des apprenants et qui ne réfèrent pas aux apprenants. Dans l'exemple suivant, le pronom « on » prend une valeur générique :

SEQ 3B :

024 Piotr et après ? + on devient un zen $\downarrow$

En effet, Piotr explique le principe de la méditation et la possibilité de devenir zen. Le pronom «on» s'applique également aux personnages de la photographie, mais pas uniquement. Dans l'extrait suivant de la séquence $1 \mathrm{~A}$, il se réfère aux spectateurs :

\footnotetext{
SEQ 1A :

191 Djibril pourquoi? parce que: d'abord ++ on n'attendait pas un mariage dans un pêle-mêle comme ça le mariage c'est dans l'église
}

Djibril, spectateur de la photographie, exprime ici sa surprise de voir un mariage sur le lieu de la photo. Le « on » pourrait aussi se référer à des témoins de la scène qui se situent dans le hors-champ, comme le photographe par exemple. Cette séquence, où les apprenants sont exposés à la photo Les mariés, est caractérisée par une instabilité énonciative, notamment dans le discours de Djibril qui emploie les pronoms « on » et « nous » :

SEQ 1A :

024 Djibril oui tout est cassé tout est en machins hein avec ça + nous avons des vies c'est-à-dire on vit + avec même situation comme ça on fait des mariages on fait des choses

026 Djibril oui même même tout est cassé tout est en machins même + on va rester toujours + la vie continue

043 Djibril il faut il faut que on être ensemble pour + compléter

084 Djibril même avec des difficultés on partage des vies

199 Djibril mais + avec tout ce pêle-mêle derrière + fumée des machins mais finalement on fait des mariages même 
Les valeurs de ces pronoms alternent entre le générique et le spécifique. En effet, à partir de la scène représentée par le photographe, Djibril interprète de façon générale le sens de la photo. Au tour de parole 024, le pronom «nous » peut référer aux personnages de la photographie. Dans ce cas, Djibril fait parler les personnages et le pronom possède une valeur spécifique. Une telle interprétation peut s'appuyer sur les déictiques " ça », " comme ça ». Cependant, le "nous » peut également revêtir une valeur générique et signifier que les hommes en général ont des vies malgré les difficultés. Les deux occurrences du pronom « on » au tour de parole 024 peuvent être interprétées de la même manière. Aux tours de parole 026, 043 et 084, la valeur générique semble l'emporter sur la valeur spécifique. Djibril propose une interprétation générale de l'image. Au tour de parole 199, en revanche, la présence de déictiques («ce», «derrière ») indique que Djibril se réfère plus particulièrement aux personnages qui font le mariage. Toutefois, avec l'emploi du pluriel pour « des mariages », on peut penser qu'il se réfère à l'ensemble de la population qui continue de vivre et de se marier malgré les difficultés. Il s'agit ici d'un exemple d'instabilité énonciative. En effet, Djibril semble utiliser le personnage pour parler de lui et exposer ses propres idées. On se situe entre la réalité et le fictionnel. Ce phénomène est évoqué par A. Baptiste et al. : « chacun est invité à se dire en disant ce qu'il voit sur la photographie» (1991: 34). Dans les extraits suivants, les apprenants naviguent aussi entre le spécifique et le générique, entre le réel et le fictionnel :

SEQ 1A :

032 Roshan parce que + perdu c'est quoi perdu c'est pas grave + mais nous restons euh + toujours euh très proches

\section{6}

\section{Roshan nous}

157 Djibril on va rester comme ça

158 Roshan nous on va nous rester très très proches

Roshan et Djibril, en faisant parler les personnages, semblent parler d'eux. Ainsi, la valeur spécifique du pronom «nous », sous couvert de se référer aux personnages, permet aux apprenants d'exprimer leur propre conception. Les apprenants en disant se disent et en voyant se voient.

On peut distinguer la valeur générique du pronom « on » de sa valeur spécifique dans l'exemple suivant :

SEQ 4A :

174 Djibril peut-être avec dix ans + vingt ans avec Roshan avec Catherine on va rencontrer un autre pays

Ici, le «on» se réfère à «je »+ «tu»+ «tu». Les allocutaires sont nommés : « avec Roshan avec Catherine ». Djibril ne se réfère pas aux personnages de la photographie. La référence n’est pas virtuelle.

Enfin, le corpus comporte des occurrences du pronom « vous » :

SEQ 4A :

102 Roshan l'autre euh + quand vous restez + toujours ensemble

248 Roshan euh + si vous voulez faire + euh + si vous voulez faire quelque chose

$249 \mathrm{P} \quad \mathrm{mhm}$

250 Roshan quelque + comme ça vous restez ensemble

$251 \quad \mathrm{P} \quad \mathrm{mmh}$

252 Roshan vous gagnez

Dans ces exemples, le pronom « vous » possède une valeur virtuelle et générique. Il permet d'impliquer les auditeurs. Les allocutaires ne sont pas les personnages de la photographie comme dans le cas de l'impératif. Ce «vous virtuel» (Cicurel 2003: 3) est évoqué par F. Cicurel: «le destinataire est virtuellement impliqué, marqué par un vous à caractère fictif» (2001 : 25). Ainsi, l'étude de la polyphonie et de la référence des pronoms personnels permet de cerner des marques de fiction. D'une part, les apprenants se réfèrent aux personnages. D'autre part, ils emploient les pronoms de façon virtuelle sans référence spécifique. 


\section{L'entrée dans la fiction}

Si la polyphonie constitue un observable pour la fiction, on peut s'interroger sur la façon dont la fiction est introduite. On considèrera tout d'abord les marques de l'irréel, avant d'envisager le discours rapporté et l'attribution de titres par les apprenants.

\subsection{Les marques de l'irréel}

F. Cicurel souligne que certains termes indiquent l'entrée dans la fiction. Il s'agit de «marqueurs de décrochements » comme «imagine, par exemple, je sais pas» (Cicurel $2003: 7$ ). Elle explique : «A tout moment, la fiction peut être introduite sous la forme d'exemples venant de l'enseignant annoncés par des "indicateurs de fictivité" (si..., par exemple..., quand..., etc.) indiquant qu'on entre dans le monde fictionnel de l'interaction » (Cicurel 1996: 84). Dans le corpus, on trouve en effet ces « indicateurs de fictivité » comme «par exemple», «quand», «si ». Cependant, ils sont introduits par les apprenants. Ainsi, «par exemple » amorce une situation-exemple :

\section{SEQ 2A :}

036 Roshan moi je par exemple par exemple moi je arrêter toi (montre avec un apprenant) VIENS après vente + pour Catherine (montre avec l'enseignante) cinquante euros ça c'est esclave

Roshan cherche à expliquer le terme «esclave » aux deux autres apprenants présents dans la classe. Il introduit son explication par «par exemple». On trouve ici des marques de la polyphonie. En effet, le locuteur Roshan, pour expliquer à ses pairs un mot, met en scène un énonciateur vendeur d'esclaves. Il désigne un apprenant par «toi », mais il se réfère à un esclave. Il emploie l'impératif «VIENS » en se référant à l'esclave. Dans la mise en scène de Roshan, l'enseignante joue le rôle du maître qui achète un esclave. Ce tour de parole met en exergue l'importance du non-verbal et de la théâtralisation.

«Quand» peut également constituer une marque d'entrée dans la fiction :

SEQ 4A :

098 Roshan euh quand vous restez toujours xxx

102 Roshan l'autre euh + quand vous restez + toujours ensemble

Roshan introduit une situation imaginaire marquée par un « vous » virtuel avec « quand ».

F. Cicurel souligne l'absence possible de marqueurs de l'irréel dans la fiction: «Mais il faut aussi constater qu'il y a fréquemment effacement des termes imaginez, inventez, car les apprenants savent ce qu'ils ont à faire devant une situation «stimulus » ou une image. Ils produisent un texte montrant qu'ils acceptent de faire comme si réellement il se passait quelque chose, comme s'il y avait un personnage qui agissait de telle ou telle manière, qui prononçait telle ou telle parole » $(1991: 266)$. Cela semble être le cas dans l'exemple suivant où Roshan introduit une hypothèse :

SEQ 1A :

147 Roshan après mariage euh + vient quel problème

$148 \mathrm{P} \quad \mathrm{mhm}$

149 Roshan euh nous restons ensemble toujours comme ça + très proches (fait un geste d'union)

\section{Roshan voilà voilà + qu'est-ce que vient quel problème est arrivé}

En effet, il ne précise pas que la situation est imaginaire par un décrocheur comme « quand », «si », «par exemple ». Cependant, le fait d'introduire une action («vient quel problème» en 147, «qu'est-ce que vient quel problème est arrivé » en 154) indique que la fiction commence. 


\subsection{Le discours direct rapporté}

De plus, le discours direct rapporté introduit la fiction dans le discours des apprenants. M.-A. Morel et L. Danon-Boileau soulignent que le discours rapporté direct occupe «une place importante dans l'oral spontané » (1998: 129) et qu' « on le rencontre évidemment dans le récit» (ibid.). Or, les apprenants s'expriment dans un oral relativement spontané. Ainsi, une situation où les apprenants commentent une photographie avec des personnages semble particulièrement propice au déclenchement du discours direct rapporté. C'est ce que remarque C. de Margerie à propos d'une photo : «Chacun pouvait imaginer ce que ces jeunes garçons se disaient » (2006:102).

O. Ducrot met en évidence la présence de plusieurs responsables, c'est-à-dire de plusieurs locuteurs, « dans les phénomènes de double énonciation [...] notamment dans le discours rapporté en style direct » (1984 : 193). Le discours direct rapporté constitue pour lui un «cas particulier de double énonciation » (1984 : 197) qui permet de faire la distinction entre sujet parlant et locuteur. Si le discours direct rapporté constitue une reprise de paroles, O. Ducrot précise cependant : «Que le style direct implique de faire parler quelqu'un d'autre, de lui faire prendre en charge des paroles, cela n'entraîne pas que sa vérité tienne à une correspondance littérale, terme à terme » (1984:199). Dans notre situation, les apprenants ne rapportent pas des paroles qui ont été émises précédemment. Ils les inventent entièrement.

Le discours direct rapporté est souvent introduit par le verbe support « dire », comme le soulignent M.-A. Morel et L. Danon-Boileau (1998 : 129). C'est le cas dans l'exemple suivant :

\section{SEQ 1A :}

082 Djibril ben je pense euh + c'est-à-dire dans le mariage + on dit je vais être fidèle à elle + même dans les situations de paix comme les situations on va partager des vies

Il s'agit d'un exemple de fiction : le «je » ne réfère pas à Djibril mais à un locuteur virtuel, par exemple le personnage de la photo. La délimitation du style direct porte à confusion ici. Deux possibilités existent. D'une part, le discours direct peut être limité au segment «je vais être fidèle à elle». Une telle interprétation est guidée par la pause : «elle + même » qui marque le changement de régime discursif. Dans ce cas, le reste de l'énoncé, «même dans les situations de paix comme dans les situations on va partager des vies ", correspond à un commentaire du discours direct rapporté qui précède. D'autre part, le discours direct peut concerner toute la deuxième partie du tour de parole : «je vais être fidèle à elle + même dans les situations de paix comme les situations on va partager des vies ». Le personnage peut en effet dire dans le même segment qu'il va être fidèle et qu'il va partager des vies. Un dernier élément est significatif dans le tour de parole de Djibril : il ne dit pas « je vais être fidèle à toi », mais «je vais être fidèle à elle ». Ainsi, avec le «je », l'apprenant se réfère au personnage de la photo, mais c'est avec la troisième personne du singulier qu'il se réfère à la future épouse du personnage. Cela constitue un exemple d'instabilité énonciative.

Dans l'exemple suivant, le discours direct rapporté n'est pas introduit par le verbe « dire » :

$$
\begin{aligned}
& \text { SEQ 1A: } \\
& 010 \text { Djibril ah (éclats de rire) ça c'est très joli euh }+ \text { vous avez fait du }
\end{aligned}
$$$$
\text { doublage des photos }+ \text { des photos mariage }+ \text { je jure euh : je }
$$$$
\text { vais être fidèle à elle bon }
$$

Cet extrait peut contenir plusieurs régimes discursifs. D’une part, «je jure» est une parole pouvant être attribuée au personnage de la photo Les mariés. D'autre part, il peut être un marqueur du discours direct « je vais être fidèle à elle». Le mot «bon» semble marquer la fin du discours direct rapporté. M.-A. Morel et L. Danon-Boileau précisent que «le début du contenu de discours rapporté est d'ailleurs luimême systématiquement marqué à l'initiale par une interjection («ah», «oh», «bah»...) ou par un ligateur (« oui », «bon», «ben», « mais »...)» (1998: 130). Dans les exemples suivants, le discours rapporté n'est pas toujours marqué par un élément autre que le verbe introducteur : 


\footnotetext{
SEQ 4A :

011 Djibril

ils veulent rencontrer mais + malgré ça + la femme $+x$ il dit non pas question il faut rester chacun sa place (rires)

031 Djibril $\mathrm{mmh}:+$ comment je vais expliquer en français ? y a des choses c'est un peu miracle + on peut pas + on peut pas le faire mais on dit + on va faire

Djibril mais on dit on va pousser c'est ça la vie
}

SEQ 4B :

142 Anna l'homme il regarde et il dit oh c'est pas possible qu'est-ce que tu fais

144 Anna et il dit sur le visage le visage il dit c'est pas possible qu'est-ce que tu fais parce que

Dans la séquence 4A, Djibril introduit le discours direct rapporté par le ligateur «non » (011). Au tour de parole 031, une pause marque le début du discours direct. Mais au tour de parole 037, le passage au discours direct est mis en évidence seulement par le verbe introducteur «dire ». Dans la séquence 4B, Anna introduit les paroles de l'homme par une interjection «oh» (142), mais ce n'est pas le cas au tour de parole 144. M.-A. Morel et L. Danon-Boileau précisent que « la démarcation finale de la séquence de DRD [discours rapporté direct] n'est pas toujours aussi aisée » (1998: 131). Effectivement, dans le discours de Djibril à la séquence 4A, la fin du discours direct rapporté n'est pas clairement délimitée. Par exemple, les segments «il fait rester chacun sa place» (011) ou «c'est ça la vie» (037) peuvent soit constituer des extraits du discours direct rapporté soit des commentaires du locuteur. Le discours direct rapporté n'est pas forcément introduit par un verbe introducteur comme dans l'exemple suivant :

$$
\text { SEQ 2A : }
$$

098 Roshan ça c'est pas prier peut-être euh viens difficile tout de suite donc maintenant oh Dieu quelque chose comme ça

Dans ce tour de parole, Roshan semble faire parler les garçons de la photo Kisangani Zaïre-Congo. La référence à la prière, même sous la forme négative, («c'est pas prier ») introduit l'exclamation «oh Dieu » qui peut être attribuée aux garçons. Cependant, l'absence d'introducteur confère à cet extrait une instabilité énonciative.

\subsection{Attribution de titre et instabilité énonciative}

Enfin, l'attribution de titres semble propice à l'entrée dans la fiction. C. de Margerie en fournit un exemple : «un étudiant - le plus politisé des étudiants du groupe - a dit : «ça c'est une réclame pour inviter les gens à voter ». Et spontanément il a proposé comme slogan : « tu votes, c'est moi qui récolterai le résultat » (2006: 102). Le slogan suggéré par l'apprenant («tu votes, c'est moi qui récolterai le résultat») constitue un exemple de fiction. Dans la séquence 3A, les apprenants ont proposé de façon spontanée une légende à la photo Nina et Julie :

SEQ 3A :

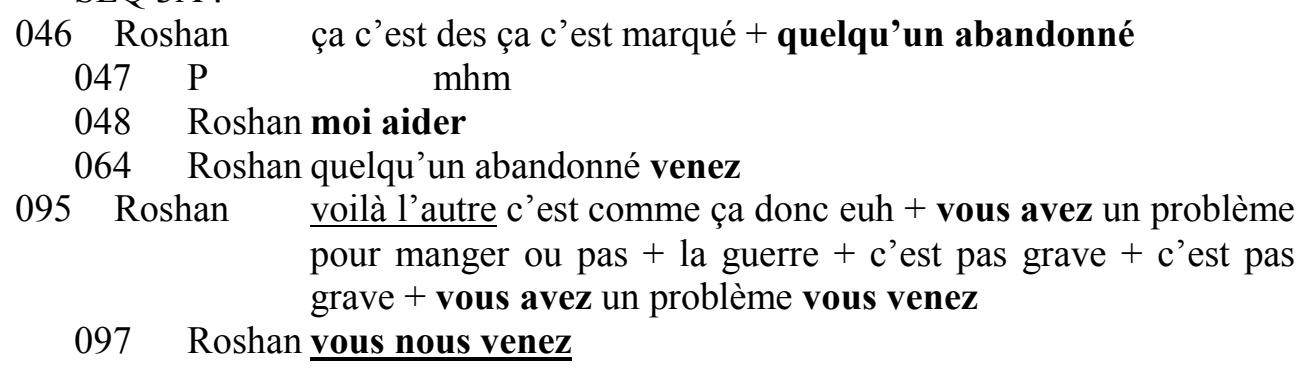




\section{Roshan l'assurance aussi comme ça + parce que ça $+*$ for your life*}

111 Roshan vous avez un problème + vous venez ? oui

Roshan énonce différents slogans : «quelqu'un abandonné moi aider» (046, 048), «quelqu'un abandonné venez » (064), « vous avez un problème vous venez» $(095,097,111)$. Ces propositions de légendes sont marquées par la virtualité des locuteurs. Dans le premier exemple («quelqu'un abandonné moi aider »), la première personne du singulier ( «moi ») peut correspondre à différents énonciateurs. Il peut s'agir notamment des spectateurs qui voient l'affiche et qui sont des donateurs potentiels. Mais le slogan aurait été rédigé par les membres de l'organisation non gouvernementale évoquée par Roshan. Cet énoncé ressemble ainsi à l'exemple d'O. Ducrot «Je, soussigné...., autorise mon fils à [...]. Signé... » (1984 : 194). Celui qui signe n'est pas l'auteur empirique du texte, mais après avoir signé, il apparaît comme le locuteur de l'énoncé.

Les autres propositions de Roshan sont marquées par l'emploi de « vous » et « nous ». Le « vous » (dans « vous avez un problème » en 095 ou en 111) correspond également aux spectateurs de l'affiche, non plus aux donateurs mais aux bénéficiaires de l'aide. Le " nous » apparaît également et il réfère aux membres de l'organisation qui aident les destinataires de l'affiche. F. Yaiche (2002: 56) souligne également la présence des pronoms «nous » et « vous » dans les slogans. Enfin, le discours de Roshan comporte un segment en anglais « for your life » en 103. L'apprenant parle à cet endroit d'une assurance qui pourrait avoir produit l'affiche. La deuxième personne du singulier («your» en 103) s'adresse toujours aux spectateurs potentiels de la publicité.

Dans la séquence 5B, les apprenants suggèrent des titres pour la photo Excited man :

$\begin{array}{lll}\text { SEQ } & \text { BB : } & \\ 354 & & \text { Rumalda mon petit ourson } \\ 358 & \text { Af } & \text { mon petit } \\ 359 & \text { P } & \text { AH il est pas + il est pas petit (rires) tu dis Viktor } \\ 360 & \text { Am } & \text { c'est rigolo } \\ 361 & \text { P } & \text { mmh ? d'autres idées de titres ? Luiza? } \\ 362 & \text { Luiza } & \text { mon doudou } \\ 363 & \text { P } & \text { mon doudou ? ouais ? } \\ 364 & \text { Carolina } & \text { mon doudou voilà c'est intéressant mon doudou }\end{array}$

Les propositions des apprenants sont marquées par la polyphonie, par exemple avec l'emploi de «mon » $(354,358,362,363,364)$. La première personne du singulier renvoie à l'énonciateur présent sur la photographie, l'homme avec la peluche. Le segment «mon doudou » est repris plusieurs fois de façon autonymique, par l'enseignante en 363, mais aussi par Carolina qui commente la proposition et la ratifie : « mon doudou voilà c'est intéressant mon doudou ». O. Ducrot souligne l'ambiguïté des reprises : « dès qu'il y a une forme de reprise (et rien n'est plus fréquent que la reprise dans la conversation), l'attribution des trois propriétés à un sujet parlant unique devient problématique - même lorsqu'il s'agit d'un énoncé syntaxiquement simple» (1984: 192). On a ici affaire à une «énonciation collective » (Cicurel 1996 : 88). F. Cicurel souligne le «glissendo énonciatif» (1996: 86) et le « dédoublement énonciatif» (1996: 87) dans le cas de reprises d'énoncés fictifs.

Ainsi, le corpus est caractérisé par une récurrence de la narration chez les apprenants. Le récit peut prendre la forme de la fiction avec virtualité des locuteurs et pseudo-référence. On se situe alors dans un exemple d'instabilité énonciative entre la réalité et le fictionnel. Ce phénomène est fréquent dans les classes de langue et concerne particulièrement les activités créatives.

\section{Les implications didactiques : fiction et créativité}

On envisagera ici les perspectives didactiques de l'activité proposée sous l'angle de la créativité. D. Coste souligne la «créativité ludique » présente dans la fiction (1991 : 247). Il existe un lien entre image et 
créativité; les photographies favorisent la construction d'univers imaginaires, permettant ainsi aux apprenants de s'exprimer.

\subsection{Le rôle des images dans la créativité}

La créativité consiste à produire sous la contrainte. Elle permet de réaliser en classe de langue des activités s'éloignant des situations de communications quotidiennes et utiles. Dans le cadre de la créativité orale, les apprenants sont amenés à se prêter à des jeux de rôle. Ils font alors parler les personnages, comme il a été montré dans notre corpus avec l'exemple de la polyphonie. D. Coste (1975: 14) souligne que l'activité de prêter des paroles aux personnages est présente dans la méthodologie SGAV. Ainsi, le discours direct rapporté, qui apparaît spontanément chez les apprenants face aux photographies, pourrait être exploité dans le cadre de jeux de rôle. F. Debyser suggère des « techniques de composition et d'écriture collective d'histoires» (1986: 111). Il est ainsi possible de "varier l'énonciation narrative» $(1986: 112)$ et «la polyphonie des structures narratives» (ibid.). Cela permettrait ainsi d'exploiter la polyphonie présente dans le discours des apprenants de façon spontanée.

F. Yaiche (2002) propose des activités guidées à partir de photographies et ne se contente pas de demander aux apprenants de verbaliser leurs impressions. La dimension créative est plus importante car l'activité comporte des contraintes. F. Yaiche suggère par exemple l'imagination de slogans par les apprenants (2002: 46). Dans le discours des apprenants de notre corpus, la création de slogans est apparue de façon spontanée sans avoir été déclenchée. Selon F. Yaiche, il est possible de « construire une histoire (seul ou en groupe) à partir d'un support projectif comme une simulation globale ou une photo » (2002 : 11). Dans son ouvrage Les simulations globales, l'auteur indique la technique du rêve éveillé dirigé. Il propose aux apprenants de « laisser vagabonder leur imagination » (1996: 116) par exemple à partir d'un support visuel comme la photographie : « les élèves feront part de leurs idées et impressions » (1996 : 117). Une telle activité peut apparaître dans le cadre des simulations globales.

L'importance de l'imaginaire dans le discours des apprenants nous conduit à envisager des activités créatives à partir des photographies. Les verbalisations à partir d'images peuvent donc constituer une étape vers la création d'activités mettant en jeu la narration, l'imaginaire et les émotions des apprenants.

\subsection{Introduction à d'autres mondes possibles grâce aux photographies}

Les photographies libèrent l'imaginaire des apprenants de telle sorte qu'ils racontent une histoire. La fiction émane des apprenants et n'est pas déclenchée par l'enseignante. F. Cicurel postule l'existence d'une compétence encyclopédique chez les apprenants : «Les événements sont interprétés selon une multiplicité de scénarios que le lecteur possède mentalement» (1991 : 266). En se référant à U. Eco, elle souligne : «Le lecteur interprète le fictionnel en fonction de son expérience du monde. Il en est de même dans la production d'énoncés fictifs dans la classe de langue » (ibid.). Ainsi, la création de la fiction chez les apprenants est liée à leur expérience.

Dans la situation analysée, les images sont souvent à l'origine de la fiction. En effet, les apprenants font parler les personnages des photos. Lorsqu'ils se réfèrent à des locuteurs virtuels, les exemples imaginés leur viennent de ce qui est représenté sur les photos. D. Coste souligne «l'appel constant à d'autres mondes possibles que celui de l'environnement immédiat et de la réalité ambiante » (1991: 247), dans les interactions en classe. Ici, les « autres mondes possibles » sont créés à partir des photographies.

L'entrée «dans ces mondes imaginaires » est mise en évidence par F. Cicurel (2003 : 10). F. Yaiche se réfère aux mondes créés avec l'expression : « univers fantasmatique » (2002: 13). En effet, grâce à la photographie, les apprenants échappent à l'environnement de la classe pour imaginer des situations en relation avec ce qui est représenté sur les images. En cela, les photos peuvent être considérées comme des moyens de communiquer en classe. F. Cicurel souligne : "Pour apprendre, il faut communiquer : or la communication ne s'établit pas de façon spontanée » (1996: 80). Dans ce cas, l'image peut figurer au titre des « outils pédagogiques » (ibid.). 
Le support des photographies sollicite les apprenants dans «leur affectivité et leur imagination» (Baptiste et al. 1991: 91). Il semble ainsi que l'image, en éveillant les affects et les émotions des apprenants, permette aux locuteurs de s'impliquer dans le discours. On suppose que cette implication déclenche une production orale plus conséquente, focalisée davantage sur le contenu que sur la forme. Pour V. Viallon, l'image peut « inciter les apprenants à s'engager entièrement (émotions, sentiments), et à être réceptifs aux interactions avec les autres » (2002: 57). Les apprenants s'expriment et se dévoilent à partir des images.

Il convient cependant de se demander dans quelle mesure l'expression des apprenants favorise l'acquisition. Ne disposant pas d'étude longitudinale, il n'est pas possible de savoir si cette activité permet l'acquisition langagière. Néanmoins, elle encourage une gestion autonome de l'interaction. Elle peut alors favoriser l'acquisition des pratiques d'interaction orale et la gestion de la conversation à bâtons rompus.

\section{Conclusion}

Ainsi, la fiction apparaît dans le discours des apprenants dès lors qu'ils emploient le « je » et le «nous » sans se référer à eux-mêmes ou le « tu » et le « vous » sans se référer à leurs pairs ou à l'enseignante. Les théories de $\mathrm{O}$. Ducrot sur la polyphonie peuvent éclairer ce phénomène de pseudo-référence et de virtualité des locuteurs. D'une part, les apprenants imaginent les paroles des personnages des photographies, mais ils se réfèrent également à des locuteurs virtuels dans des situations-exemples imaginaires. La mise en scène et la théâtralité présentes dans le discours des apprenants semblent propices à des activités créatives qui permettent le recours à des univers imaginaires. Il importe de souligner que la fiction apparaît à l'initiative des apprenants. Ainsi, le récit n'est pas déclenché par des questions de l'enseignante mais par la nature des photos. Cette expérience peut constituer un prélude à l'élaboration d'activités créatives à partir de photographies.

\section{Références bibliographiques}

Baptiste A., Belisle C., Pechenart J.-M., Vacheret C. (1991) Photolangage. Une méthode pour communiquer en groupe par la photo. Paris : Les éditions d'organisation.

Beacco J.-C. (2007) L'approche par compétences dans l'enseignement des langues. Paris: Didier, Langues et didactique.

Cicurel F. (1985) Parole sur parole ou le métalangage dans la classe de langue. Paris : Clé international, Collection Didactique des langues étrangères.

Cicurel F. (1991) «L'identité discursive d'un apprenant en langue» in Russier C., Stoffel H., Véronique D. (éd.) Interactions en langue étrangère. Aix-en-Provence : Publications de l'université de Provence, pp. 259-269.

Cicurel F. (1996) «L'instabilité énonciative en classe de langue: du statut didactique au statut fictionnel du discours » in Cicurel F., Blondel E. (éd.) La construction interactive des discours de la classe de langue. Paris : Les Carnets du CEDISCOR 4, Presses de la Sorbonne nouvelle, pp. 77-92.

Cicurel F. (2001) "Quand le français langue professionnelle est l'objet de l'interaction » in Cicurel F., Doury M. (éd.) Interactions et discours professionnels, usages et transmission. Paris: Les Carnets du CEDISCOR 7. , Presses de la Sorbonne nouvelle, pp. 21-36.

Cicurel F. (2002) «La classe de langue un lieu ordinaire, une interaction complexe». AILE, 16. [En ligne: http://aile.revues.org/document801.html].

Cicurel F. (2003) "Les réagencements contextuels». Marges linguistiques [En ligne: http://www.revuetexto.net/marges/marges/Documents\%20Site\%206/doc0137_cicurel_f/doc0137.pdf].

Coste D. (1975) «Les piétinements de l'image ». Etudes de linguistique appliquée, NS, 17 (janvier-mars 1975), « L'image en didactique des langues » (sous la coordination de Coste D.). Paris : Didier. pp. 5-28. 
Didactique et enseignement, français langue maternelle, français langue seconde DOI $10.1051 / \mathrm{cmlf0} 8049$

Coste D. (1991) «Le fictionnel ordinaire des discours d'apprenants » in Russier C., Stoffel H., Véronique D. (éd.) Interactions en langue étrangère. Aix-en-Provence : Publications de l'université de Provence, pp. 245- 248.

Debyser F. (1986) « Production de textes et matrices narratives ». Pratiques, $n^{\circ}$ 50, pp. 111-114.

Ducrot O. (1984) Le dire et le dit. Paris : Les Editions de Minuit.

Ducrot O. et al. (1980) Les mots du discours. Paris : Collection Le sens commun, Les éditions de Minuit.

Larcher P. (1998) «Le concept de polyphonie dans la théorie d'Oswald Ducrot » in Vion R. (éd.) Les sujets et leurs discours. Enonciation et interaction. Aix-en-Provence : Publications de l'Université de Provence, pp. 203-224.

Margerie C. de (2006) Entretien privé in Muller, C. (2007) L'image comme déclencheur de l'expression. Analyse des interactions en classe face à des photographies, Annexes, mémoire de master 2 recherche, Université Paris 3 , pp.101-114.

Morel M.-A., Danon-Boileau L. (1998) Grammaire de l'intonation. L'exemple du français. Paris : Bibliothèque de faits de langues, FDL, Ophrys.

Soares Da Rocha D. O. (1996) «La fiction dans le cadre de l'interaction didactique : une lecture du théâtre de Ionesco » in Cicurel F., Blondel E. (éd.) La construction interactive des discours de la classe de langue. Paris : Les Carnets du CEDISCOR 4, Presses de la Sorbonne nouvelle, pp. 61-71.

Viallon V. (2002). Images et apprentissages, le discours de l'image en didactique des langues. Paris : L'Harmattan.

Yaiche F. (1996) Les simulations globales, mode d'emploi. Vanves: Hachette livre Français langue étrangère.

Yaiche F. (2002) Photos-Expressions. Paris : Pratiques de classe, Hachette Français langue étrangère.

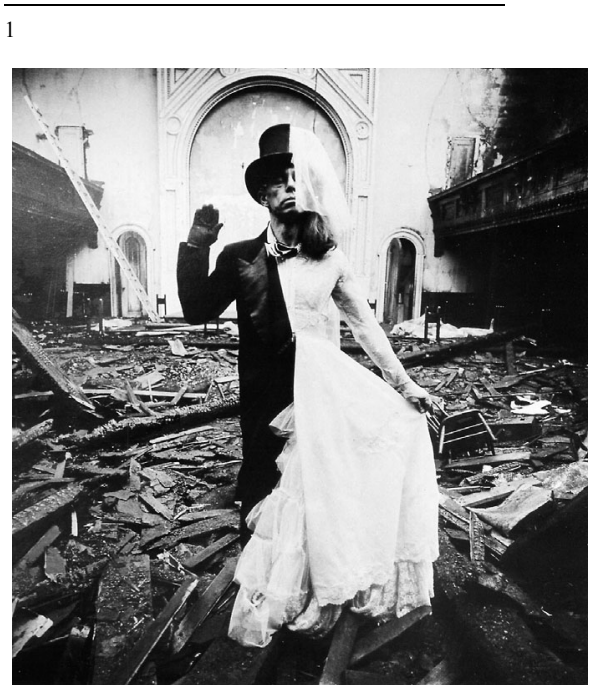

Les mariés

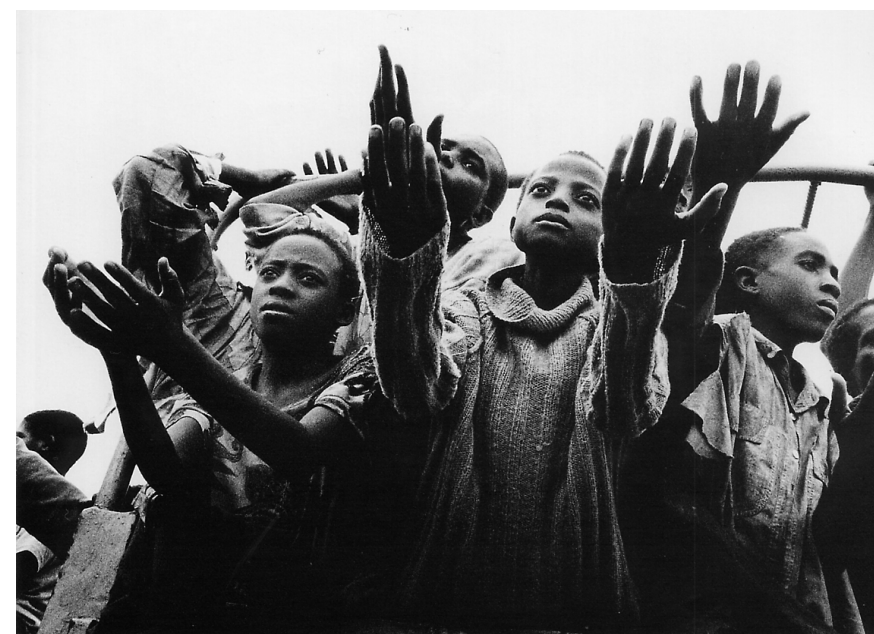

Kisangani, Zaïre- Congo 


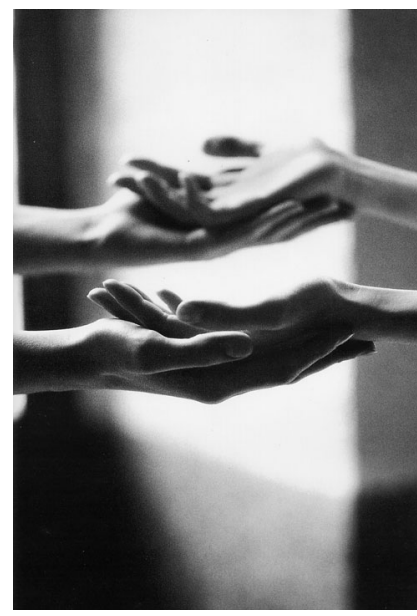

Nina et Julie

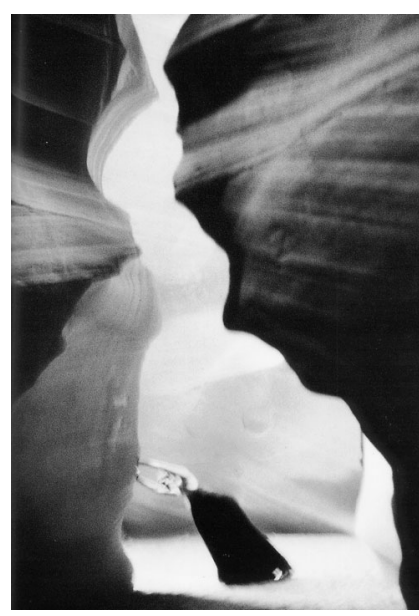

Honor Frazer

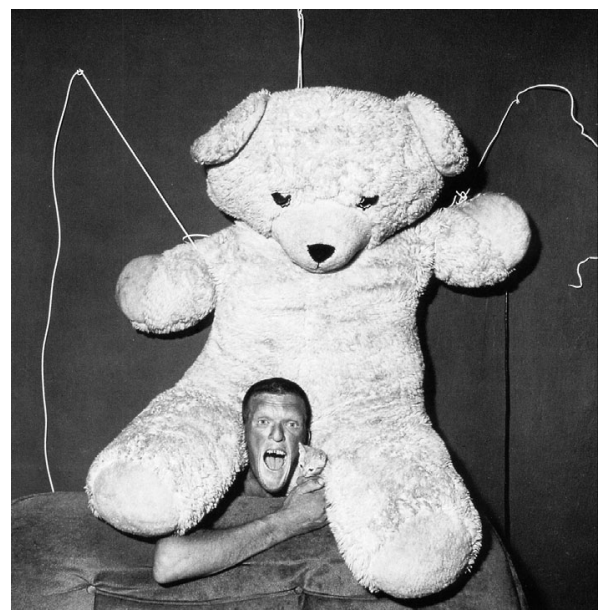

Excited man

${ }^{2}$ Conventions de transcription :

xxx segment inaudible; (rires) commentaire sur le non-verbal ; : allongement de la syllabe ; + pause ; + + pause plus longue $;+++$ pause au-delà de 5 secondes $;$ ? intonation montante $; \downarrow$ intonation descendante $;. .$. demande d'achèvement; AH OUI accentuation; maintenant chevauchement; $* *$ langue autre que langue-cible; P professeur ; Magda prénom de l'apprenant quand identifié ; Af non identifié (femme) ; Am non identifié (homme) ; A ? apprenant non identifié. Les prénoms des apprenants ont été modifiés en tenant compte de leur sexe et de leur nationalité. Le prénom réel de l'enseignante, quand il est employé par les apprenants, a été conservé. 